\title{
Monitoring of fresh-cut spinach leaves through a multispectral vision system
}

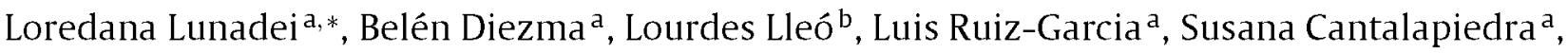 \\ Margarita Ruiz-Altisent ${ }^{\mathrm{a}}$
}

${ }^{a}$ Laboratorio de Propiedades Físicas y Tecnologias Avanzadas en Agroalimentaciôn, Departamento de Ingeniería Rural, ET.S.I. Agrônomos, Universidad Politécnica de Madrid, Av. Complutense s/ $n$, Ciudad Universitaria, 28040 Madrid, Spain

b Departamento de Ciencia y Tecnologías Aplicadas a la Ingenieria Técnica Agricola, E.U.I.T. Agricolas 5, Universidad Politécnica de Madrid, Av. Complutense s/n, Ciudad Universitaria, 28040 Madrid, Spain

Keywords:

RTU leafy spinach

Shelf life

Multispectral image

Image algorithm

Classification

\begin{abstract}
A B S T R A C T
This paper reports the development of image processing methods for the detection of superficial changes related to quality deterioration in ready-to-use (RTU) leafy spinach during storage. The experiment was performed on spinach leaves stored at $4.5^{\circ} \mathrm{C}$ for 21 days (Set 1 ) and at $10^{\circ} \mathrm{C}$ for 9 days (Set 2). Regarding Set 1,75 units were evaluated beginning at time zero and after 7,14 , and 21 days of storage (treatments $t_{1.0}, t_{1.1}, t_{1.2}$, and $t_{1.3}$, respectively). In the case of Set 2,24 samples were measured at time zero and after 3,6 , and 9 days (treatments $t_{2,0}, t_{2,1}, t_{2,2}$, and $t_{2,3}$, respectively). Multispectral images were acquired using a 3-CCD camera centered at the infrared (IR), red (R), and blue (B) wavelengths. Opportune combinations of these bands were calculated using virtual images, and a non-supervised classification was performed. A large number of spinach leaves belonging to Set 2 showed injuries due to the effects of in-pack condensation; thus, an image algorithm was developed to separate these defective leaves before applying the classification. For Set 1 , Set 2 and all the calculated virtual images, the classification procedure yielded two image-based deterioration reference classes (DRCs): Class A, including the majority of the samples belonging to $t_{1.0}$ and $t_{1.1}$ (Set 1 ) and to $t_{2.0}$ and $t_{2.1}$ (Set 2 ) treatments and Class $B$, which comprised mainly the samples belonging to $t_{1.2}$ and $t_{1.3}$ (Set 1) and to $t_{2.2}$ and $t_{2.3}$ (Set 2) treatments. An internal validation was performed, and the best classification was obtained with the virtual images based on $\mathrm{R}$ and $\mathrm{B}$ bands. For each sample, camera classification was evaluated according to reference measurements (visible (VIS) reflectance spectra and CIE $L^{*} a^{*} b^{*}$ coordinates); in all cases, VIS reflectance values corresponded well with storage days, and Classes $A$ and $B$ could be considered homogenous with regards to $L^{*}$ and $a^{*}$ values. Taken together, these results confirmed that a vision system based on $R$ and $B$ spectral ranges could constitute an easy and fast method to detect deteriorating RTU packed spinach leaves under different refrigeration conditions.
\end{abstract}

\section{Introduction}

Consumption of ready-to-use (RTU) vegetables has been increasing in recent years, especially as a result of changes in the lifestyles of consumers (Ragaert et al., 2004). However, processing of vegetables promotes faster physiological deterioration, biochemical changes, and microbial degradation of the product. These phenomena may result in the development of strong unpleasant odors, decay, discoloration, and tissue softening (Watada and Qi, 1999). For this reason, most RTU products are sold within a few weeks after packaging.

Postharvest techniques for preserving the quality of fresh-cut vegetables are aimed at maintaining external color and inter-

\footnotetext{
* Corresponding author. Tel.: +34 913365862; fax: +34913365845.

E-mail address: loredana.lunadei@gmail.com (L. Lunadei).
}

nal phytonutrient content, preventing tissue degradation and improving hygiene. Disorders arising from processing can be minimized by the use of sharp cutting tools, enzymatic browning inhibitors, modified atmospheres and low temperature. The most frequently used temperature is $4^{\circ} \mathrm{C}$, which is considered optimal for many leafy vegetables. This temperature permits a slow down of plant metabolic processes, which helps to prevent chilling injury (Jacxsens et al., 2002). In spite of this, published data suggest that none of the available washing and sanitizing methods can guarantee the microbiological quality of minimally processed vegetables without compromising their sensory quality. Regarding RTU baby spinach, the major problems are the development of a strong unpleasant odor and discoloration (Allende et al., 2004). Because consumers usually purchase fresh-cut products based on their visual appearance, color is extremely important (Kader, 2002; Ferrante et al., 2004). RTU vegetables can undergo color changes due to different biochemical processes involving 
plant pigments that are important for leaf function. For this reason, variations in pigment content (e.g., carotene and chlorophyll degradation and oxidation of phenolic compounds) may provide information concerning the physiological state of leaves. Plant pigment content can be evaluated through traditional methods (e.g., chemical extraction), which are time-consuming, labor intensive and require destruction of the samples; thus, these methods do not permit measurement of changes in pigments over time for a single sample. However, plant pigments can be estimated quickly through non-destructive techniques such as measurement of spectral reflectance. A large number of spectral indices have been developed for the estimation of leaf pigment content. In the visible (VIS) spectral range, most of these indices employ wavelengths belonging to the red (R, 660-700 nm), blue (B, 450-480 nm) and green bands ( $G, 520-560 \mathrm{~nm}$ ) because chlorophyll, carotenoids and anthocyanins absorb particularly strongly in these regions (Sims and Gamon, 2002; Xue and Yang, 2009). In the infrared region of the spectrum, the near-infrared (NIR) is the most useful for the estimation of chlorophyll content. By combining IR and R, two major indexes have been developed: the well known normalized difference vegetation index (NDVI), which is IR $-R / I R+R$ (Gitelson and Merzlyak, 1996; Zude, 2003), and the R/IR index (Bodria et al., 2004; Lleó et al., 2009). However, spectrometers and colorimeters can analyze only small portions of the product and thus require the repetition of the analysis in different areas of the product. Additionally, they can only be used to evaluate objects with uniform surfaces; non-uniform surfaces may be blended or processed to provide uniformity, but this will only provide an average color and will not represent the original appearance of the sample (Abbott, 1999). Computer vision systems allow quantification and classification based on the original color and appearance of the sample, even if the samples are of varying size, shape, or texture (Studman, 2001). For these reasons, it could be interesting to estimate the leaf pigment content through a multispectral vision system based on the cited sensitive bands.

Multi-spectral images usually contain two or more monochrome images. Each monochrome image contains the amount of light reflected off the objects in the picture for a particular spectral band. Many food processing industries have adapted computer technology to assess the quality of products such as fruit, cheese, and shrimps (Coles et al., 1993; Tunde and Feldoldi, 2000; Allende et al., 2004). With the use of an image capture device such as a video or a digital camera, an image can be analyzed by application of the appropriate algorithms. A variety of techniques related to multi-spectral images have been used for a multitude of purposes (Kranzier, 1985; Park and Chen, 2001; Hahn, 2002; Borhan et al., 2004; Kleynen et al., 2005; Zheng et al., $2006 a, b)$. The aim of this work was to classify RTU spinach leaves on the basis of changes in quality during storage by employing a multispectral vision system. The main objective was to identify proper virtual images, as combinations of monochromatic images, to detect changes in color that were related to deterioration in quality.

\section{Materials and methods}

\subsection{Spinach samples}

Spinach (Spinacia oleracea) used in this project was RTU leafy spinach, packed in sealed plastic bags $(200 \mathrm{~g})$ characterized by an inside atmosphere of $3-6 \% \mathrm{O}_{2}, 0-0.5 \% \mathrm{CO}_{2}$ and $93.5-97 \% \mathrm{~N}_{2}$. A single leaf of spinach was considered as one sample unit for these experiments. Two sets of experiments were conducted on two sets of samples. In the first experiment, packed leafy spinach obtained from an orchard in Murcia, Spain, was stored at $85 \% \mathrm{RH}$ and $4.5^{\circ} \mathrm{C}$
(Set $1, n=300$ ), which was considered the optimal storage temperature. In the second experiment, a second set of samples (Set 2, $n=96$ ) consisting of packed leafy spinach purchased from a local wholesale produce distributor was stored at $10^{\circ} \mathrm{C}$ for 9 days to reproduce the most frequent domestic storage conditions. Regarding Set 1, seventy-five units were evaluated per day: at time zero (treatment $t_{1.0}$ ) and after 7 (treatment $t_{1.1}$ ), 14 (treatment $t_{1.2}$ ) and 21 (treatment $t_{1.3}$ ) days of storage. Regarding Set 2, twenty-four samples were measured at time zero (treatment $t_{2.0}$ ) and after 3 (treatment $t_{2.1}$ ), 6 (treatment $t_{2.2}$ ), and 9 (treatment $t_{2.3}$ ) days. Because Set 1 and Set 2 were submitted to distinct treatments, different analyses were performed on each set.

\subsection{Reference values: visible reflectance spectra and color parameters}

By employing a portable spectrophotometer (Minolta Sensing Inc., CM-50I, Japan), visible (VIS) relative reflectance spectra (360-740 nm, at $10 \mathrm{~nm}$ intervals) were obtained and $C I E L^{*} a^{*} b^{*}$ color coordinates were measured, where $L^{*}$ is the luminance component (ranging from 0 to 100 ), and $a^{*}$ and $b^{*}$ are color coordinates related respectively with the red/green and yellow/blue spectral ranges, with values varying from -120 to +120 (Yam and Papadakis, 2004). The colorimeter measurement area had a diameter of $8 \mathrm{~mm}$, and all measurements were taken under the conditions of standard illuminant D65 and $10^{\circ}$ observer. A standard white calibration plate was employed to calibrate the equipment. Measurements were performed three times on one side of every spinach leaf by positioning the measurement area near the center of the samples while avoiding the central leaf venation. An average VIS relative reflectance spectrum and a set of color coordinates, expressed as average values, were thus obtained for each sample. For each sample, VIS relative spectra and color parameters were considered reference parameters and were compared with the storage days and with the camera classification. All analyses were performed using MatLab ${ }^{\circledR}$ software (MathWorks Inc., Natick, MA, USA).

\subsection{Vision systems}

Digital images, comprised of 1300-by-1000-by-3 data arrays (IRRB images, which defined the infrared, red, and blue color components for each individual pixel), were acquired through a multispectral imaging system consisting of a frame-grabber (National Instruments ${ }^{\circledR}$, Austin, TX, USA) and a 3-CCD camera (DuncanTech/Redlake MS-3100 ${ }^{\circledR}$, Redlake Inc., USA) with a digital output. The camera was equipped with three band-pass filters (bandwidth: $20 \mathrm{~nm}$ ) centered at $800 \mathrm{~nm}$ (infrared, IR), $680 \mathrm{~nm}$ (red, R), and $450 \mathrm{~nm}$ (blue, B). A light source was provided by six $100 \mathrm{~W} / 220 \mathrm{~V}$ halogen lamps, and the object distance between the lens system and the sample was $60 \mathrm{~cm}$ (Fig. 1). The angle between the camera lens axis and the lighting source axis was $45^{\circ}$ because the diffuse reflection responsible for the color occurs at $45^{\circ}$ from the incident light (Francis and Clydesdale, 1975; Marcus and Kurt, 1998). The images were acquired using a black background. A black canvas was put around the vision test station to create a uniform light field around the object.

A second type of digital image was acquired from the samples with a Nikon D80 ${ }^{(\mathbb{B})}$ digital camera (Nikon Corp., Tokyo, Japan); these images were stored as 2600-by-3800-by-3 data arrays (RGB images), defining the red, green and blue components of the pixels. The RGB camera consisted of a single-lens reflex digital camera with a focal length of $50 \mathrm{~mm}$. A hemispheric cap with white reflecting walls was constructed to eliminate any effect of environmental light. At equidistant points on the inside of the cap, four $150 \mathrm{~W}$ incandescent lamps were attached. The camera was adjusted to a vertical position, and the lens was $61 \mathrm{~cm}$ above the object of 

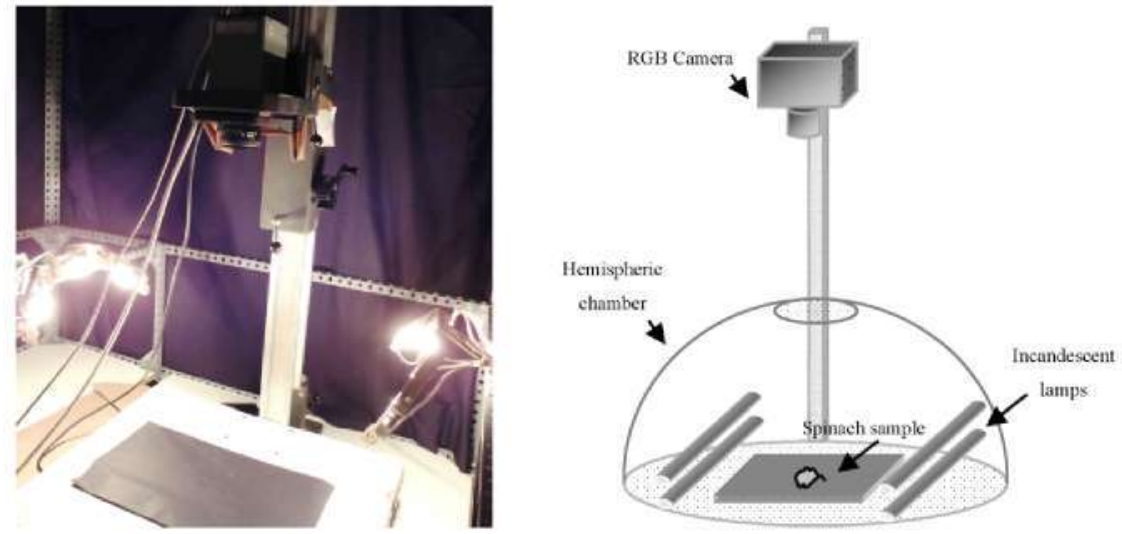

Fig. 1. The experimental IRRB vision system (on the left) and a schematic diagram of the RGB vision system (on the right).

interest. The images were acquired using a white-squared black background. Fig. 1 shows a diagram of the described RGB vision system.

\subsection{Image segmentation and virtual image calculation}

IRRB images were acquired for each sample and for each treatment, yielding 396 images (Set 1: 300 images, Set 2: 96 images). IRRB images acquired by the camera were stored and processed

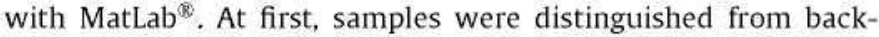
ground using the Otsu method (Otsu, 1979), which is a common segmentation technique that computes the threshold level based on the image histogram distribution. This was performed on the IR images because they presented the greatest difference between the gray levels corresponding to samples (the region of interest, ROI) and those corresponding to the background. This operation resulted in a binary image that could be considered an "image mask" where the gray level of the ROI was set to one and the gray level of the background was set to zero. This mask was multiplied by the images acquired at $800 \mathrm{~nm}(\mathrm{IR}), 680 \mathrm{~nm}(\mathrm{R}), 450 \mathrm{~nm}$ (B) and by the following virtual images: $R / I R$ ( $R$ divided by $I R),(I R-R) /(I R+R)$ (corresponding to the NDVI), $B / R$ ( $B$ divided by $R$ ) and $(R-B) /(R+B)$ (in the following: $\mathrm{R}-\mathrm{B} / \mathrm{R}+\mathrm{B})$. The first and the second virtual images were originally developed for the assessment of crop growth conditions, whereas the last two image combinations were chosen to emphasize the possible changes in the image intensity related to the red band (660-700 $\mathrm{nm}$ ), which corresponds to the spectral region of one of the absorbance peaks of chlorophyll, and to the blue region ( $450-480 \mathrm{~nm}$ ), where carotenoids and anthocyanins absorb particularly strongly. Further analyses were performed on the ROIrelative histograms of those virtual images, which were computed as the relative frequency of pixels over the intensity range of the image. In the rest of this document, "histogram" refers to "relative histogram" of the image.

\subsection{Image processing of Set 2 samples: detection of in-pack condensation}

In the second experiment, a large number of spinach leaves (especially those in the last two treatments) showed injuries in their vegetative tissue due to the effects of in-pack condensation, probably due to storage at improper temperatures $\left(10^{\circ} \mathrm{C}\right)$. The presence of these kinds of leaves could have negatively affected the following analysis because spinach affected by condensation

Table 1

Representative leaves, belonging to Set 2 and subjected to the treatments $t_{2,0}, t_{2,1}, t_{2,2}$, and $t_{23}$, with and without injuries due to in-pack condensation.

\begin{tabular}{|c|c|c|c|c|}
\hline Treatments & $t_{2,0}$ & $t_{2,1}$ & $t_{2,2}$ & $t_{2,3}$ \\
\hline \multicolumn{5}{|l|}{$\begin{array}{l}\text { Images of leaves } \\
\text { without injuries }\end{array}$} \\
\hline $\begin{array}{l}\text { Images of leaves } \\
\text { exhibiting } \\
\text { injuries }\end{array}$ & & & & \\
\hline
\end{tabular}


a) $R G B$ image

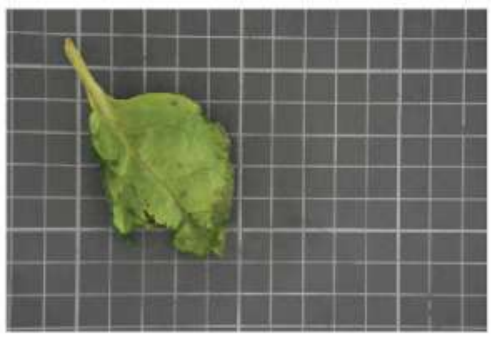

c) $G$ image

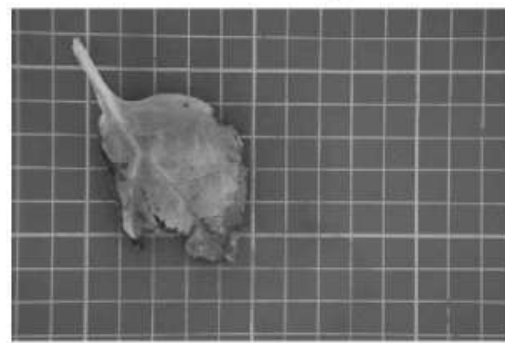

b) $S$ image

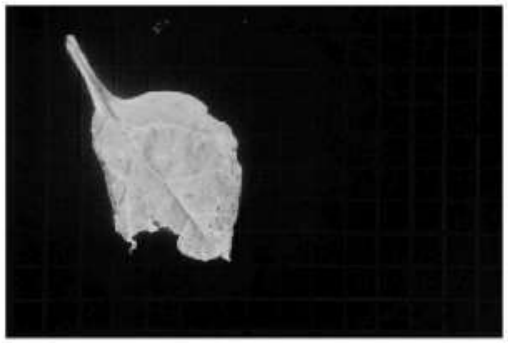

d) $S_{b w}$ image

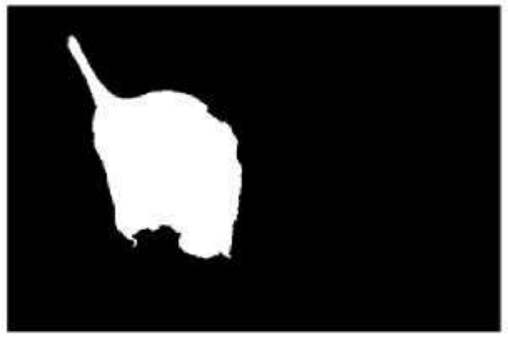

e) $\left(S_{b w} * G\right)$ image

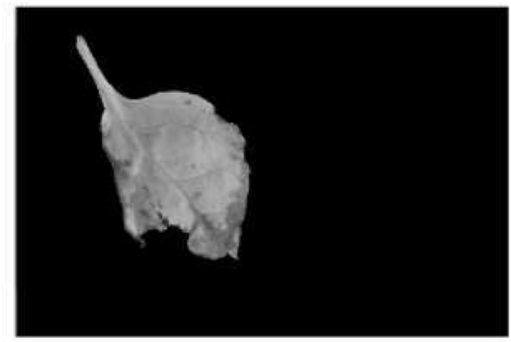

Fig. 2. The segmentation process applied to Set 2 samples: (a) RGB image of an RTU spinach leaf exhibiting injuries due to in-pack condensation; (b) $S$ image (HSV model); (c) $G$ image (RGB model); (d) $S_{b w}$ ( $S$ binary image); (e) $\left(S_{b w}{ }^{\circ} G\right)$ image (result of multiplying the $S_{b w}$ image by the $G$ image).

turned a darker green, whereas leaves that did not present this defect had reduced green pigment and increased yellow coloration. This is observable in Table 1, which shows an example of two kinds of leaves (with and without injuries due to condensation) belonging to Set 2 and subjected to the $t_{2.0}, t_{2.1}, t_{2.2}$ and $t_{2.3}$ treatments. In this table, the leaf representing the treatment $t_{2.0}$ is the same for both cases because at zero time there were no samples with injuries.

In order to consider only the leaves that had comparable surface conditions, RGB images were employed to develop an image algorithm that was able to identify and separate leaves exhibiting defects due to condensation. The first objective of the imaging process was to distinguish leaves (ROIs) from the white-squared background. Different segmentation methods were tested, and the best results were obtained after converting the RGB images to HSV format (hue, $H$, saturation, $S$, and brightness, namely the value, $V$ ) by employing MatLab ${ }^{*}$ in-core functions. The $S$ canal of the HSV images, which presented the greatest difference between the gray level corresponding to ROI and to the squared background, was binarized, obtaining a binary image $\left(S_{b w}\right) \cdot S_{b w}$ was then multiplied by the $G$ image, which presented the greatest difference between the gray level of the regions of the leaf tissue with and without injuries, thus obtaining a $\left(S_{b w}{ }^{*} G\right)$ image. The second objective was to develop an image algorithm that was able to automatically identify the $\left(S_{b w}{ }^{*} G\right)$ images of samples affected by condensation. An unsupervised pattern recognition analysis of the histograms of all the $\left(S_{b w}{ }^{*} G\right)$ images was thus performed by principal component analysis (PCA), with the aim of reducing the original number of variables ( $K=256$ intensity levels) to a much smaller number $(A)$. These variables are referred to as the principal components (PCs), which are orthogonal linear combinations of the original variables and account for most of the variability in the data. The examination of loading plots generated by PCA helps to identify the main differences between leaves affected by injuries and leaves without defects; these differences correspond to the intensity levels exhibiting the higher loading values. To test the ability of PCA to capture the variability between the different kinds of leaves, a discriminant analysis (DA) with two levels in the categorical variable (leaves with and without injuries) was performed on the intensity values of the $\left(S_{b w}{ }^{*} G\right)$ images corresponding to the intensity levels selected through PCA (i.e., intensity levels with the highest loadings). After classifying samples as leaves with and without injuries, the IRRB images of the latter were processed as explained.

\subsection{Non-supervised image classification}

For both samples sets, a non-supervised classification according to Ward's method (Ward, 1963) was performed to define deterioration reference classes (DRCs) based on histograms of the virtual images. In both cases, a multidimensional space was considered, where each dimension corresponded to an intensity level of the R/IR, NDVI, B/R, and R-B/R + B histograms. Each histogram was thus represented as a single point in a multidimensional space. Ward's classification method was applied by computing the 
a) $R G B$ image

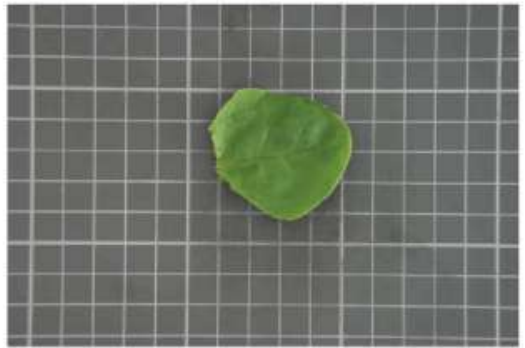

c) $G$ image

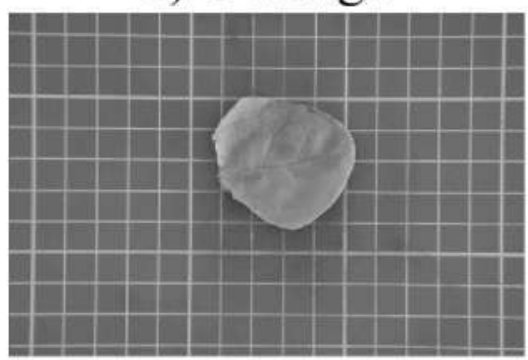

b) $S$ image

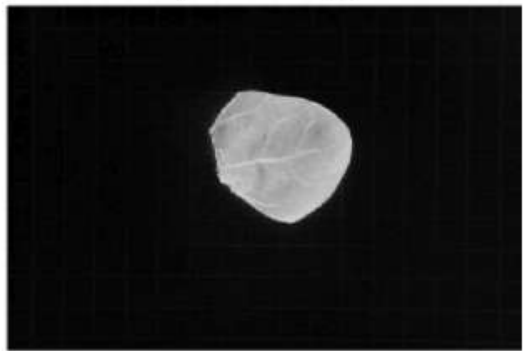

d) $S_{b w}$ image

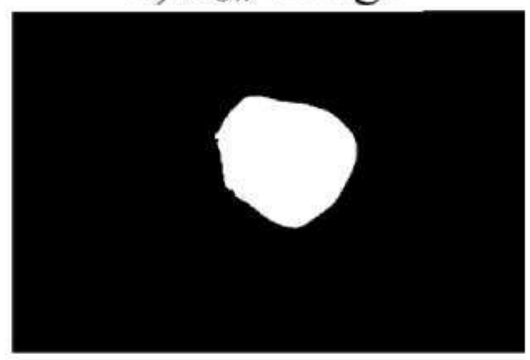

e) $\left(S_{b w} * G\right)$ image

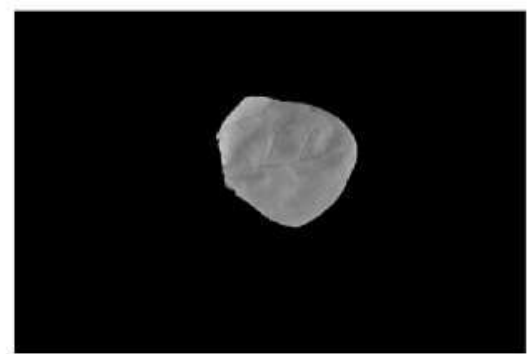

Fig. 3. The segmentation process applied to Set 2 samples: (a) RGB image of an RTU spinach without any injuries; (b) $S$ image (HSV model); (c) $G$ image (RGB model); (d) $S_{b w}$ ( $S$ binary image): (e) $\left(S_{l i w}{ }^{*} G\right)$ image (result of multiplying the $S_{b w}$ image by the $G$ image).

matrix of Euclidean distances between each pair of individuals (histograms), grouping the closest individuals and hierarchically merging groups (or individuals) whose combination gave the smallest Ward linkage distance (that is, the minimum increase within the sum of squares of the newly formed group). Ward's method is preferable to other classification methods because it takes into account all histograms of the data set at every level of the grouping, thus producing very well-structured and homogeneous groups (Otto, 2007). Additionally, this method gave rise to successful results in previous work investigating fruit ripeness and browning (Lleó et al., 2009; Herrero et al., 2011; Lunadei et al., 2011). A MatLab ${ }^{\circledR}$ devoted code was developed to automatically generate groups on the basis of the input maximum Ward linkage distance, which is derived from the analysis of the cluster tree features. An average histogram was computed for each group and defined as a DRC.

\subsection{Validation: classification of anonymous samples into deterioration reference classes}

For both experiments, internal validations were performed. The same population generating the model was classified again, one by one, into the previously generated DRCs. Essentially, the observed classification of the samples was compared with the predicted classification of the same samples; these were obtained by computing the Euclidean distances $\left(E_{d}\right)$ between the histograms of the sample images and the average histograms of the generated DRCs.

\subsection{Statistical analysis of reference values}

VIS reflectance spectra and color parameters (CIE $L^{*} a^{*} b^{*}$ coordinates) were compared to the classification based on the histograms of the virtual images. An ANOVA was performed on $L^{*}, a^{*}$ and $b^{*}$ values and on the classes extracted from the image analysis. A mean comparison procedure (LSD test) was applied with a 0.05 significance level. Statistical procedures were performed using MatLab $7.0^{\circledR}$ and STATGRAPHICS Plus 5.1 (Manugistics Inc., Rockville, MD, USA).

\section{Results and discussion}

\subsection{Detection of leaves with injuries due to condensation}

All tests confirmed that the imaging process performed on the RGB images of the Set 2 samples allowed for good image segmentation, producing images with a high difference between gray values of the leaf and of the background. Figs. 1 and 2 report the results of the proposed process in leaves with and without injuries, respectively. In the resulting $\left(S_{b w}{ }^{*} G\right)$ images, the background was black (gray level $=0$ ), the leaf tissue was light gray and possible regions with injuries were darker gray (gray level $\neq 0$ ). 

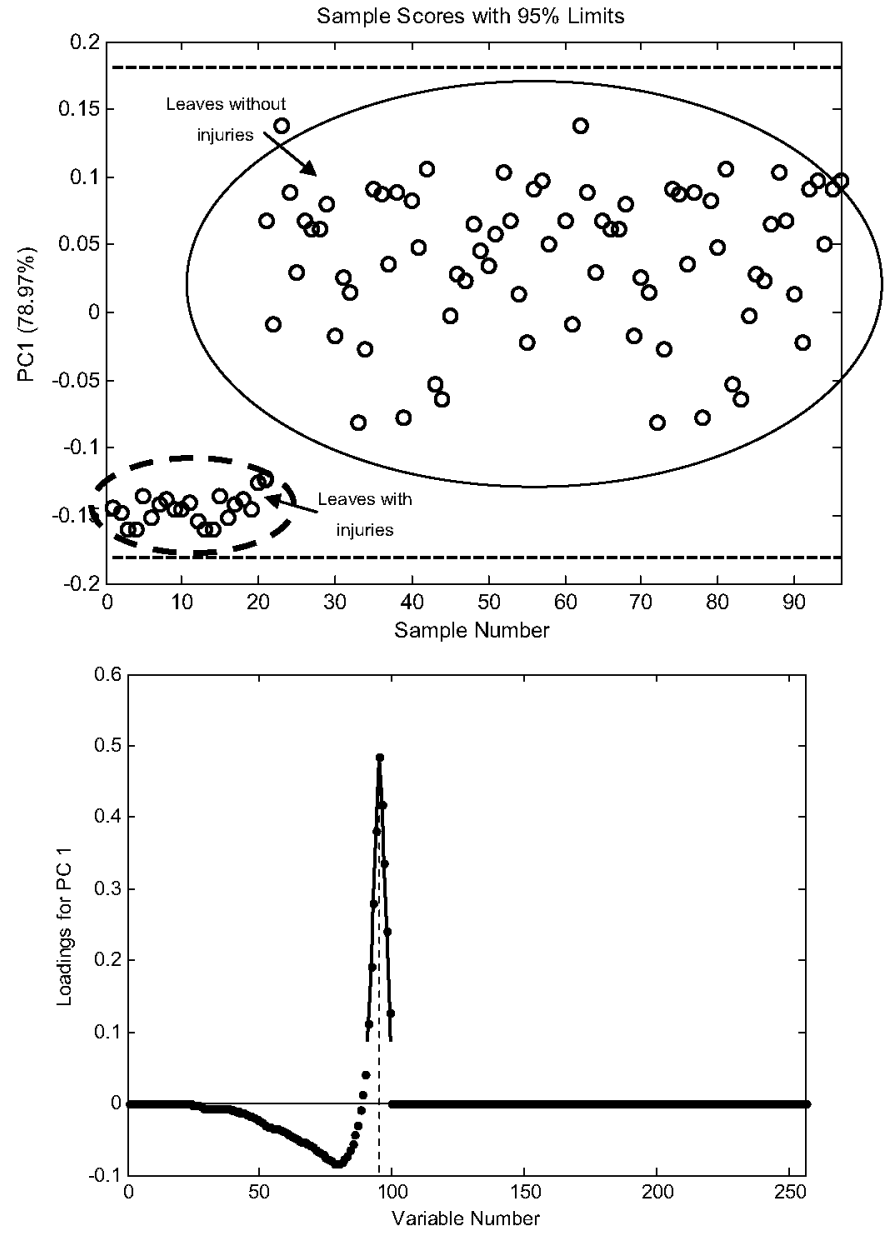

Fig. 4. Upper panel: PC1 score plot for histograms of $\left(S_{b w}{ }^{*} G\right)$ images with $95 \%$ confidence limits (dotted lines). The $X$ axis corresponds to the sample number and the $Y$ axis to the sample scores for PC1. The two individuated groups, corresponding to the samples with lower and higher PC1 scores, are marked with dotted and continuous lines, respectively. Lower panel: PC1 loadings plot for histograms of $\left(S_{b w}{ }^{*} G\right)$ images. The $X$ axis corresponds to the intensity levels $(n=256)$ and the $Y$ axis to the loading values for PC1. The dotted vertical line represents the intensity level corresponding to the maximum of the PC1 loadings.

Regarding PCA results, in the plot of PC1 scores against sample number $(n=96)$ (Fig. 3 ), samples fell into two major groups: one characterized by lower PC1 scores and another by higher PC1 scores. The two groups corresponded respectively to samples that were affected by injuries and to samples that did not have defects. This suggested that the variability of the PC1 scores, which accounted for nearly $79 \%$ of the total variance, was related to the presence of injuries and could explain the difference between the two kinds of leaves. Because PC1 yielded highly positive loadings in the 91-98 intensity levels (Fig. 3), this intensity range was related to the variability between the different kinds of leaves. Fig. 4 shows the intensity values corresponding to the two intensity levels with the highest PC1 loadings (95 and 96). This figure graphically confirms that in the mentioned range, it is possible to clearly separate leaves with and without injuries. The discriminant analysis, performed on the intensity values that ranged from 91 to 98 of the $\left(S_{b w}{ }^{*} G\right)$ images, appeared to perform a very good classification because only two samples ( $1.5 \%$ of the original cases) were not correctly classified into one of the two categorical variables (leaves with and without injuries). Through the explained algorithm, the $\left(S_{b w}{ }^{*} G\right)$ images were classified into two groups: the first one comprised leaves with injuries ( $n=19$ ), which corresponded to samples

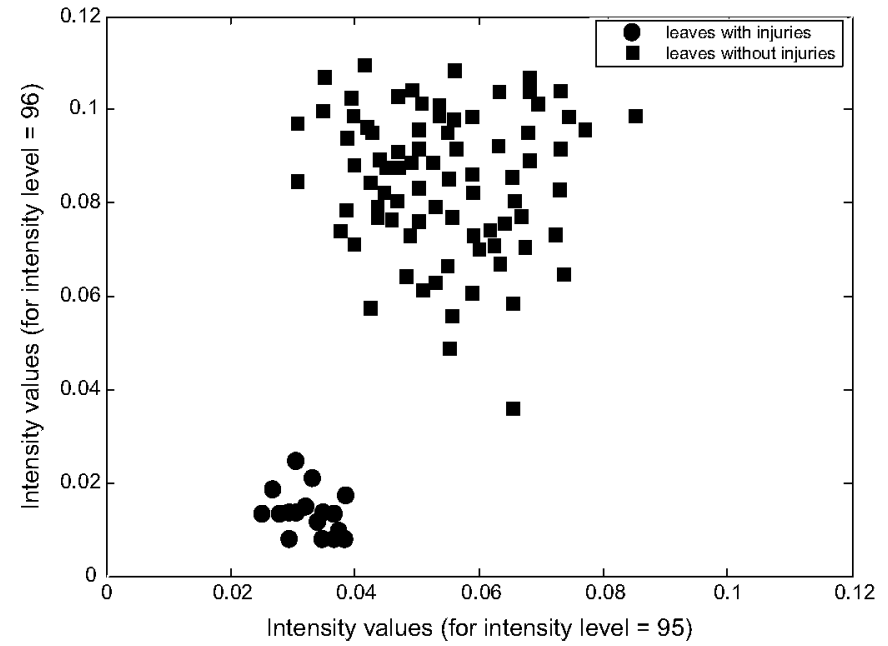

Fig. 5. Intensity values for the 95 th ( $X$ axis) and the 96 th ( $Y$ axis) intensity levels for all the $\left(S_{b w}{ }^{*} G\right)$ images of Set 2. Circles and squares refer to samples with and without injuries, respectively.

subjected to the two last treatments, and the second one was comprised of leaves without defects $(n=77)$.

\subsection{Virtual images}

Fig. 5 illustrates virtual images obtained by computing the R/IR, NDVI, $B / R$, and $R-B / R+B$ virtual images from four Set 1 samples, each one subjected to a different treatment (from $t_{1.0}$ to $t_{1.3}$ ). In $R / I R$ and $R-B / R+B$ images, the pixel intensity values increased during the storage period, whereas in the NDVI and B/R images, the pixel intensity value decreased. These changes in color did not occur uniformly in the analyzed samples; there were regions within the same samples in which pixels changed to higher (or lower) intensity values faster than others. This could be related to the increase in enzymatic activity resulting from tissue disruption at cut surfaces, according to the local substrate composition and the amount of enzyme that initiated the discoloration process. In the case of Set 2 samples, similar results were found (data not shown).

\subsection{Generation of deterioration reference classes}

The average histograms of Set 1 samples were calculated for each treatment and for each image combination (Fig. 6). In all cases, the average histograms corresponding to the first and second treatments ( $t_{1.0}$ and $t_{1.1}$ ) were quite similar and appeared separated from the average histograms of the images acquired on the 14th and 21 st day. Similar results were obtained with the Set 2 samples (data not shown), where the average histograms corresponding to the $t_{2.0}$ and $t_{2.1}$ treatments were similar and separated from those of the $t_{2.2}$ and $t_{2.3}$ treatments. Cluster analysis results regarding the Set 1 samples are graphically reported as dendrograms (Fig. 6) that confirm the above observation; by setting the maximum Ward Linkage distance within groups at 0.6 pixel relative frequency for Set 1 samples (and at 0.3 pixel relative frequency for Set 2 samples, data not shown), two clusters corresponding to two DRCs (Class A and Class B) were obtained for each virtual image. Table 2 reports the classification matrix obtained by applying cluster analysis to each image combination of samples from Set 1 . In Table 2 The nonsupervised classifications, based on the $B / R$ and $R-B / R+B$ virtual images, were able to segregate quite well between the storage periods; in both cases, almost $93 \%$ of Class A was comprised of samples measured at time zero and after seven days of storage, and almost $98 \%$ of Class B was comprised of samples analyzed on the 14 th and 
a) IRRB

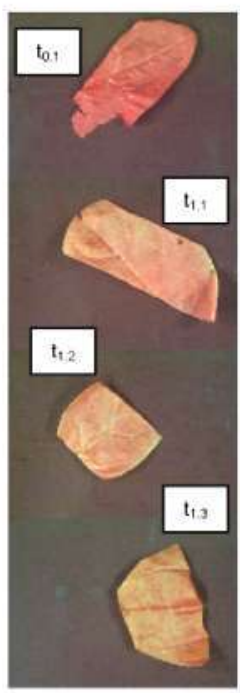

b) $R / I R$

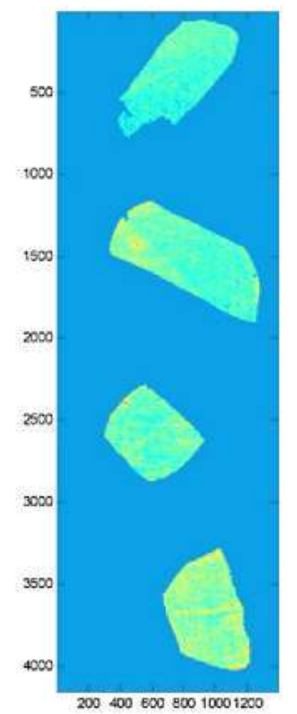

c) NDVI

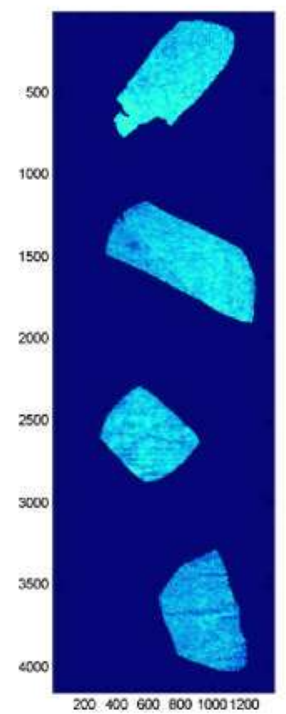

d) $B / R$

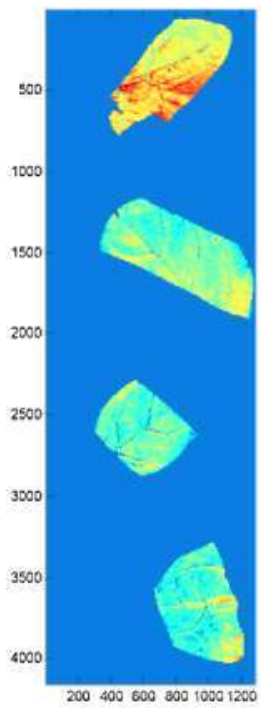

e) $R-B / R+B$

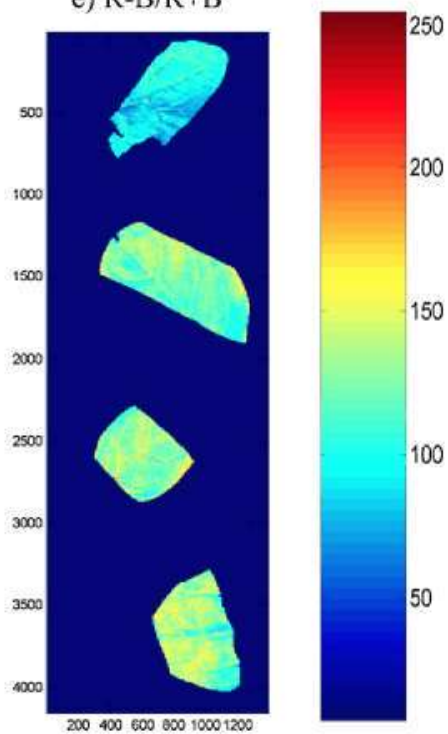

Fig. 6. IRRB (a) and virtual images (b: R/IR; ; NDVI; d: B/R; e: R-B/R+B) of a spinach leaf belonging to Set 1 calculated at time zero $\left(t_{1,0}\right)$ and after storage for 7,14 and 21 days $\left(t_{1,1}, t_{1,2}\right.$ and $\left.t_{1,3}\right)$. The color scale represents the intensity values of the images, (For interpretation of the references to color in the figure caption, the reader is referred to the web version of the article.)

21st days. However, the classifications based on the R/IR and NDVI did not segregate well between the storage periods; Class A comprised $67 \%$ (R/IR images) and $77 \%$ (NDVI images) of the samples analyzed during the first and the second treatments ( $t_{1.0}$ and $\left.t_{1.1}\right)$, and Class B comprised 80\% (R/IR images) and 76\% (NDVI images) of samples analyzed during the $t_{2.2}$ and $t_{2.3}$ treatments. The classification results regarding Set 2 samples were quite similar (data not shown) because $B / R$ and $R-B / R+B$ images exhibited the best results (near $75 \%$ of Class $A$ was composed of samples analyzed on the first and second day of treatment, and almost $70 \%$ of Class B was composed of samples analyzed on the 6th and 9th days), whereas the classifications based on the R/IR and NDVI images were not as accurate (Class A included approximately $30 \%$ and $60 \%$ of samples analyzed at time zero and after three days of storage, and Class B comprised $45 \%$ and $70 \%$ of samples analyzed on the last two days of treatment). Taken together, these results illustrate the feasibility of the proposed classification for segregating extreme stages of storage. For Set 1 , significant changes in quality took place after seven days of storage, whereas for Set 2, these changes occurred after the third day of storage. These results are similar to those obtained in another study that investigated the acceptability and shelf life of RTU lettuce by digital image analysis (Zhou et al., 2004). Zhou et al. found that, in samples stored at $4{ }^{\circ} \mathrm{C}$ and at $10^{\circ} \mathrm{C}$, the most significant changes in color occur within the first 4-6 days of storage, although the color changes of the samples stored at $4^{\circ} \mathrm{C}$ were much less dramatic than those observed at $10^{\circ} \mathrm{C}$.

\subsection{Internal validation}

In the case of $B / R$ and $R-B / R+B$ image histograms of Set 1 samples, approximately $94 \%$ and $97 \%$ of the sample images were classified in the same group by both the Ward's non-supervised classification and the classification according to Euclidean distances to Ward-generated reference classes. For R/IR and NDVI image histograms, the two methods classified approximately $85 \%$ of samples in the same group. Regarding Set 2 samples, the internal validation results obtained with $B / R$ and $R-B / R+B$ histograms were also quite satisfactory. Approximately $92 \%$ and $95 \%$ of the samples were classified in the same group by the non-supervised classification and by the classification according to Euclidean distances to the reference classes. For R/IR and NDVI histograms, approximately $83 \%$ and $84 \%$ of samples were classified in the same group by both methods (data not shown).

\subsection{Reflectance spectra and color parameters of image based clusters}

Fig. 7 shows average VIS reflectance spectra obtained from Set 1 samples at time zero $\left(t_{1.0}\right)$ and after treatments $t_{1.1}, t_{1.2}$ and $t_{1.3}$, along with the spectra obtained from Set 2 samples subjected to the $t_{2.0}, t_{2.1}, t_{2.2}$, and $t_{2.3}$ treatments. Regarding the Set 1 samples, the trend of the spectra along the storage period showed a clear general decrease in reflectance values over the spectral range. Additionally,

Table 2

Classification matrix of Set 1 spinach samples: number of samples subjected to $t_{1.0}$ and $t_{1.1}$ treatments $\left(n_{t_{1,0}-t_{1.1}}=150\right)$ and to $t_{1.2}$ and $t_{1.3}$ treatments $\left(n_{f_{1.2}}-t_{1.3}=150\right)$ (observed classification) and classified by the camera into Class A or B (predicted classification).

\begin{tabular}{|c|c|c|c|c|}
\hline & \multirow[t]{2}{*}{ Virtual images } & \multirow[t]{2}{*}{ Classes/treatments } & \multicolumn{2}{|c|}{ Observed classification } \\
\hline & & & $t_{1,0}-t_{1,1}$ & $t_{1,2}-t_{1,3}$ \\
\hline \multirow{8}{*}{ Predicted classification } & \multirow[t]{2}{*}{$R / I R$} & A & 128 & 62 \\
\hline & & B & 22 & 88 \\
\hline & \multirow[t]{2}{*}{ NDVI } & A & 114 & 33 \\
\hline & & B & 36 & 117 \\
\hline & \multirow[t]{2}{*}{$\mathrm{R} / \mathrm{B}$} & A & 146 & 12 \\
\hline & & B & 4 & 138 \\
\hline & \multirow[t]{2}{*}{$\mathrm{R}-\mathrm{B} / \mathrm{R}+\mathrm{B}$} & A & 148 & 13 \\
\hline & & B & 2 & 137 \\
\hline
\end{tabular}




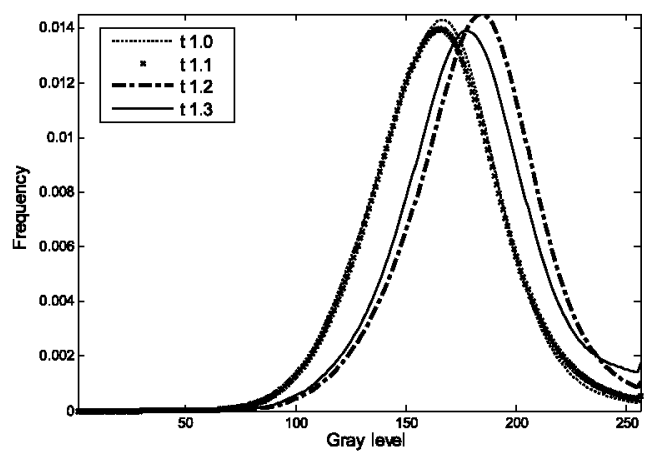

a
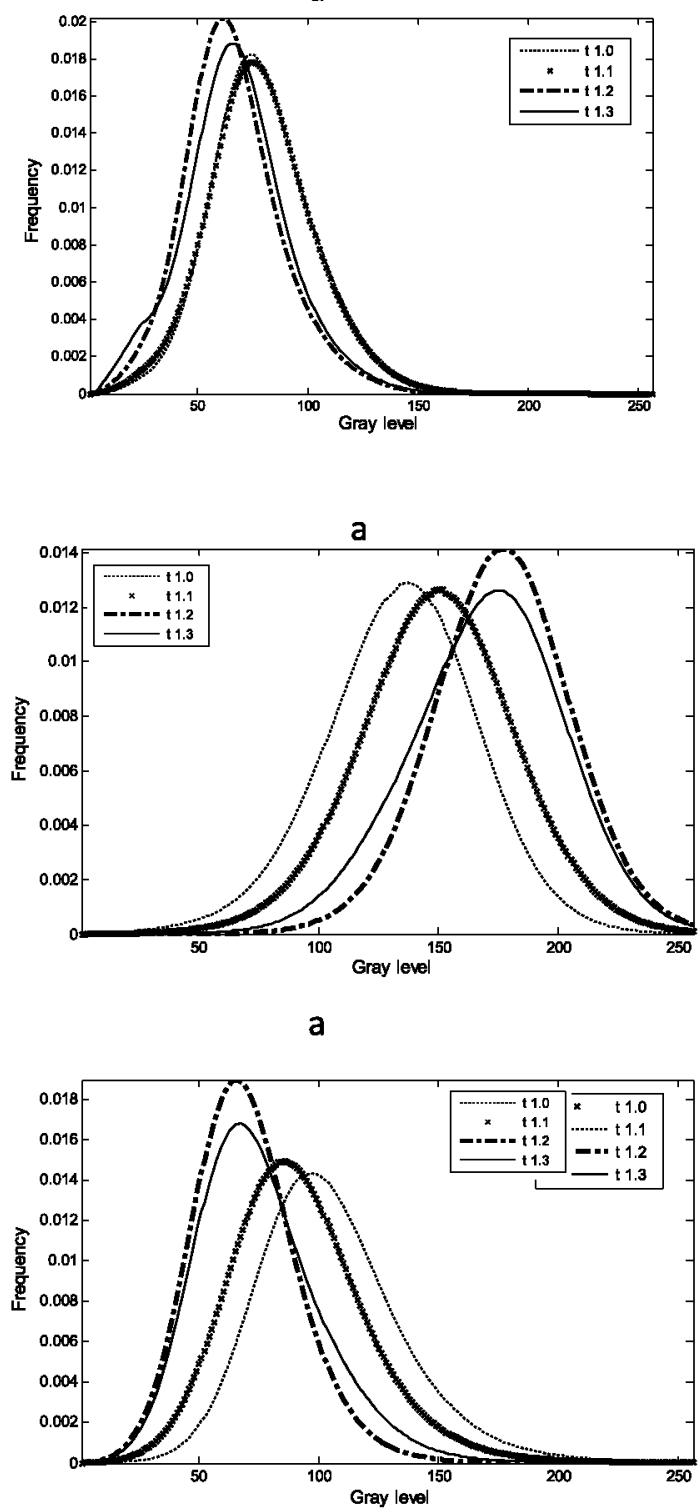

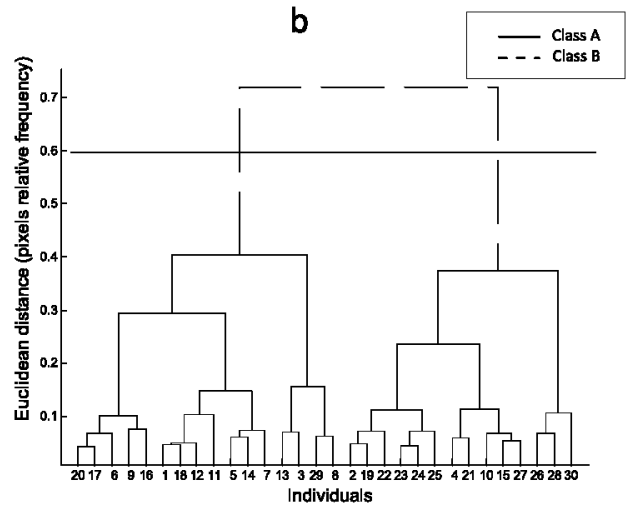

$\mathbf{R} / \mathbf{R}$

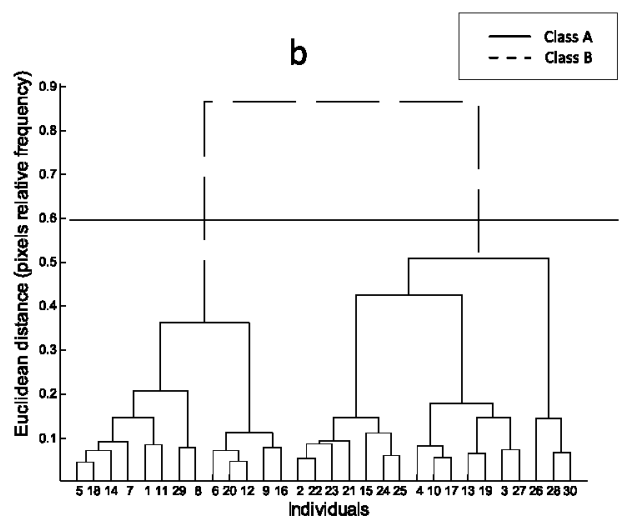

NDVI

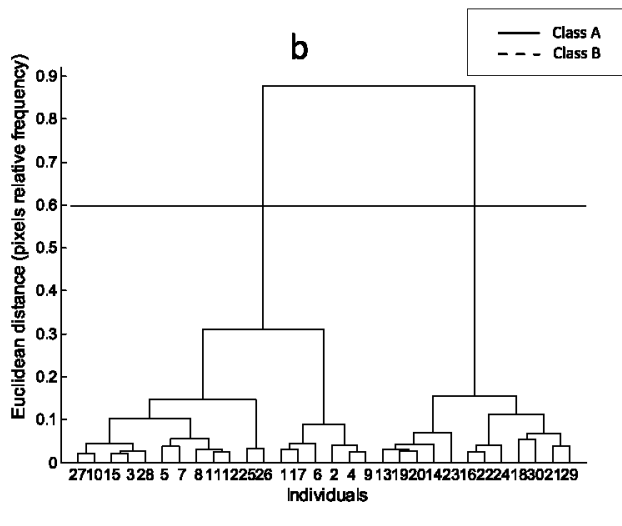

b

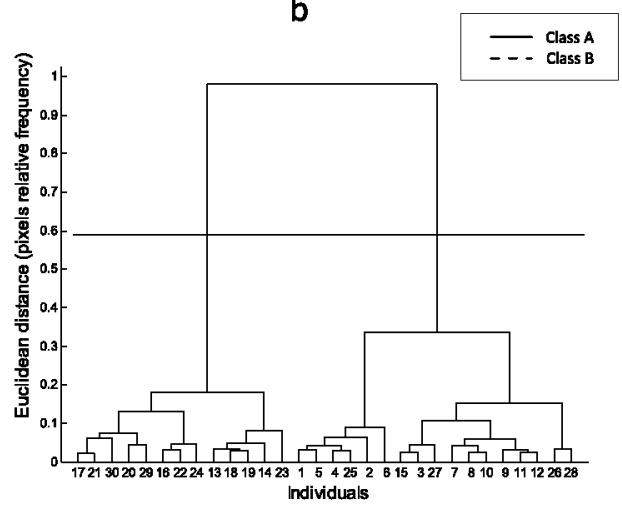

$R-B / R+B$

Fig. 7. (a) $S$ plot of the average histograms calculated from the Set 1 samples for each image-based class (R/IR, NDVI, B/R and R $-B / R+B)$ and for each treatment $\left(t_{1.0}, t_{1.1}\right.$, $t_{1,2}$, and $\left.t_{1,3}\right)$; (b) the relative dendrograms generated by applying Ward's non-supervised classification. Horizontal lines in the cluster trees represent the maximum Ward Linkage distance within groups (pixel relative frequency $=0.60$ ). 
Table 3

Average values, 95\% confidence intervals and results of the ANOVA Fisher LSD test performed on $L^{*}, a^{*}$ and $b^{*}$ values of Set 1 samples grouped in Classes A and B according to Ward's method based on $R / I R$, IR - R/IR + R, B/R, and R $-B / R+B$. An asterisk indicates that there is a significant difference $(p<0.05)$ between all of the means, whereas n.s. implies there is no significant difference.

\begin{tabular}{|c|c|c|c|c|c|c|c|}
\hline & \multirow[t]{2}{*}{ Cluster } & \multicolumn{6}{|c|}{ Color coordinates } \\
\hline & & $L^{*}$ mean & $\pm 1.96 \mathrm{SD}_{L^{*}}$ & $a^{*}$ mean & $\pm 1.96 \mathrm{SD}_{a^{*}}$ & $b^{*}$ mean & $\pm 1.96 \mathrm{SD}_{b^{*}}$ \\
\hline \multirow{3}{*}{$\mathrm{R} / \mathrm{IR}$} & $A$ & 43.41 & 0.22 & -10.86 & 0.06 & 21.58 & 0.21 \\
\hline & $\mathrm{B}$ & 47.20 & 0.43 & -8.98 & 0.08 & 21.79 & 0.22 \\
\hline & $F$-values & $12.57^{*}$ & & $15.50^{*}$ & & $3.45_{\mathrm{n} . \mathrm{s} .}$ & \\
\hline \multirow{3}{*}{ NDVI } & A & 43.63 & 0.24 & -10.46 & 0.06 & 21.02 & 0.23 \\
\hline & B & 47.57 & 0.26 & -8.99 & 0.07 & 21.28 & 0.25 \\
\hline & $F$-values & $13.06^{*}$ & & $14.14^{*}$ & & $3.49_{n, s .}$ & \\
\hline \multirow{3}{*}{$\mathrm{B} / \mathrm{R}$} & A & 42.25 & 0.18 & -11.13 & 0.06 & 21.01 & 0.23 \\
\hline & B & 48.04 & 0.20 & -8.72 & 0.07 & 21.77 & 0.22 \\
\hline & $F$-values & $27.84^{*}$ & & $23.13^{*}$ & & $3.89_{\mathrm{n}, \mathrm{s}}$ & \\
\hline \multirow{3}{*}{$R-B / R+B$} & A & 42.30 & 0.22 & -11.15 & 0.05 & 21.08 & 0.21 \\
\hline & $\mathrm{B}$ & 49.00 & 0.23 & -8.98 & 0.08 & 21.79 & 0.23 \\
\hline & $F$-values & $29.54^{*}$ & & $22.33^{*}$ & & $3.80_{\mathrm{n}, \mathrm{s}}$ & \\
\hline
\end{tabular}

the spectra corresponding to the first and second treatments $\left(t_{1.0}\right.$ and $t_{1.1}$ ) were quite similar and appeared separated from those obtained during the $t_{1.2}$ and $t_{1.3}$ treatments. In the Set 2 samples, there was a minor decrease in the reflectance values with the treatments. Additionally, the separation between the first and the second treatment as well as between the third and the fourth treatment was not as appreciable. However, in both cases the main difference was that the relative reflectance values in the green area $(520-560 \mathrm{~nm})$ were higher at the beginning of the storage period compared to the end. This indicates that the most important color changes in spinach leaves are due to the decay of the green color component. This evidence was confirmed by subsequent analysis performed on the color parameters. The observed minor decrease in the reflectance values of Set 2 compared with those of Set 1 , along with the more similar shape of the average spectra between the sets across different treatments were probably due to the different storage conditions. At $4^{\circ} \mathrm{C}$, samples exhibited a major decay in surface color (from green to yellow), whereas at $10^{\circ} \mathrm{C}$, samples were affected by condensation but the decay of the green component was not as appreciable. This evidence could explain why the results obtained through the camera classification, the objective of which was to detect changes in color surface, were not as precise compared to those obtained with Set 1 (Fig. 8).

The shape and trend of the average VIS reflectance spectra of the two cluster, $\mathrm{A}$ and $\mathrm{B}$, confirmed the above observation: in the case of Set 1, the average spectra of the clusters exhibited a clear decrease in reflectance values for all the virtual images, whereas in the case of Set 2, the two average spectra were much more similar (data not shown).

Tables 3 and 4 report the average values and 95\% confidence intervals of $L^{*}, a^{*}$ and $b^{*}$, as well as the clusters obtained through the described non-supervised classification of the image histograms calculated from each Set 1 and Set 2 sample. In both sample sets, for each virtual image and image-based cluster, an increase was observed in $L^{*}, b^{*}$ and $a^{*}$, indicating a change in black to white $\left(L^{*}\right)$, blue to yellow $\left(b^{*}\right)$, and green to red $\left(a^{*}\right)$ in samples from Classes $\mathrm{A}$ to $B$. The trend of these parameters agreed with the results of previous studies that examined the evolution of color coordinates in spinach leaves during storage (Klockow and Keener, 2009). Table 3 and Table 4 also report the results of an ANOVA Fisher Least Significant Differences (LSD) test performed on the color of the samples classified into Classes A and B by Ward's method. In all cases, the two classes were characterized by a consistent increase in $L^{*}$ and $a^{*}$ values from Class A to B, whereas $b^{*}$ values were not significantly different between the classes. Additionally, the LSD test results confirmed the previous observation regarding the decay of the green color component because $a^{*}$ values of samples from Set
1 increased more from Class A to B than the same values of samples from Set 2 . In almost all the cases, $a^{*}$ values showed the best agreement between clusters. These results indicate that the proposed classification was able to select the proper classes (Classes A and $B$ ) that could be considered homogenous with regard to color parameters. Considering the four image-based classifications, in both sets of samples, $B / R$ and $R-B / R+B$ images showed the best agreement between cluster classification and color parameters. On the basis of these results, image-based classes may provide relevant
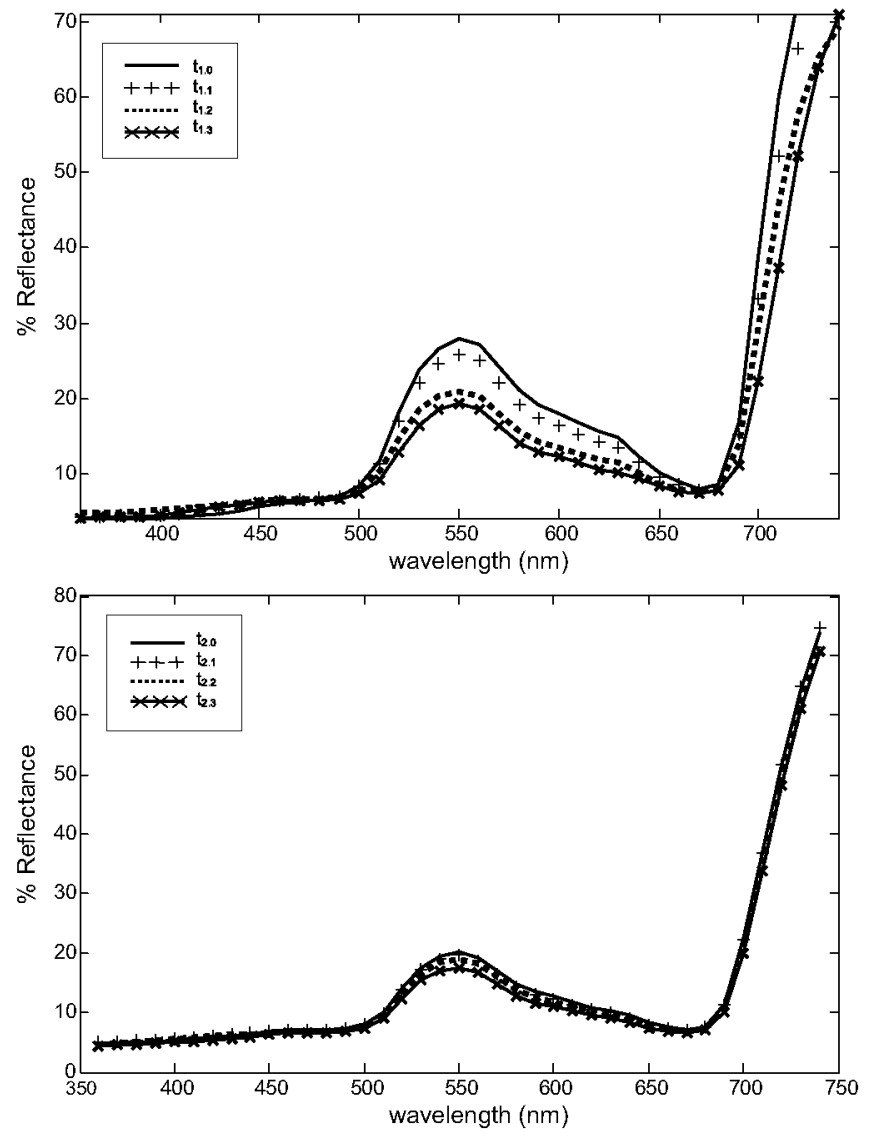

Fig. 8. Average VIS reflectance spectra obtained from Set 1 samples after treatments $t_{1.0}, t_{1.1}, t_{1.2}$, and $t_{1.3}$ (upper panel) and from Set 2 samples after treatments $t_{2.0}, t_{2.1}$, $t_{2.2}$, and $t_{2.3}$ (lower panel). The $X$ axis corresponds to the wavelength (nm) and the $Y$ axis to the VIS relative reflectance of the spectra (arbitrary units). 
Table 4

Average values, 95\% confidence intervals and results of the ANOVA Fisher LSD test performed on $L^{*}, a^{*}$ and $b^{*}$ values of Set 2 samples grouped in Classes A and B according to Ward's method based on $R / I R$, IR $-R / I R+R, B / R$, and $R-B / R+B$. An asterisk indicates that there is a significant difference ( $p<0.05)$ between all of the means, whereas $n . s$. implies there is no significant difference.

\begin{tabular}{|c|c|c|c|c|c|c|c|}
\hline & \multirow[t]{2}{*}{ Cluster } & \multicolumn{6}{|c|}{ Color coordinates } \\
\hline & & $L^{*}$ mean & $\pm 1.96 \mathrm{SD}_{L^{*}}$ & $a^{*}$ mean & $\pm 1.96 \mathrm{SD}_{a^{*}}$ & $b^{*}$ mean & $\pm 1.96 \mathrm{SD}_{b^{*}}$ \\
\hline \multirow{3}{*}{$\mathrm{R} / \mathrm{IR}$} & $A$ & 40.20 & 0.12 & -10.71 & 0.06 & 21.88 & 0.21 \\
\hline & $\mathrm{B}$ & 44.41 & 0.13 & -10.28 & 0.08 & 22.19 & 0.22 \\
\hline & $F$-values & $14.18^{*}$ & & $13.98^{*}$ & & $3.55_{\mathrm{ns} .}$ & \\
\hline \multirow{3}{*}{ NDVI } & A & 40.57 & 0.14 & -11.40 & 0.06 & 21.08 & 0.23 \\
\hline & B & 44.63 & 0.16 & -10.99 & 0.07 & 21.33 & 0.25 \\
\hline & $F$-values & $13.67^{*}$ & & $13.83^{*}$ & & $3.8_{\mathrm{n}, \mathrm{s} .}$ & \\
\hline \multirow{3}{*}{$\mathrm{B} / \mathrm{R}$} & A & 40.04 & 0.18 & -10.55 & 0.06 & 21.24 & 0.23 \\
\hline & $\mathrm{B}$ & 45.45 & 0.20 & -9.72 & 0.07 & 22.20 & 0.22 \\
\hline & $F$-values & $16.60^{*}$ & & $16.63^{*}$ & & $3.83_{\mathrm{n}, \mathrm{s}}$ & \\
\hline \multirow{3}{*}{$R-B / R+B$} & $A$ & 40.00 & 0.13 & -11.01 & 0.05 & 21.60 & 0.21 \\
\hline & $\mathrm{B}$ & 45.70 & 0.12 & -9.98 & 0.08 & 22.79 & 0.23 \\
\hline & $F$-values & $16.77^{*}$ & & $17.43^{*}$ & & $3.97_{\text {n.s. }}$ & \\
\hline
\end{tabular}

information for the management of RTU spinach leaves and have the potential to detect the most significant changes in surface color.

\section{Conclusions}

In the present study, a new method based on a multispectral vision system was proposed to classify RTU leafy spinach exposed to two different storage temperatures $\left(4^{\circ} \mathrm{C}\right.$ and $\left.10^{\circ} \mathrm{C}\right)$ on the basis of color changes, which were related to quality deterioration. An image algorithm was also developed to automatically identify and separate leaves exhibiting injuries due to the effects of in-pack condensation. The classification method utilized relative histograms of four virtual images (R/IR, NDVI, B/R, and R - B/R+B) computed as combinations of infrared (IR, $800 \mathrm{~nm}$ ), red ( $R, 680 \mathrm{~nm}$ ) and blue (B, $450 \mathrm{~nm}$ ) monochromatic images. Specifically, R/IR and NDVI employed the IR and $R$ spectral ranges, whereas $B / R$ and $R-B / R+B$ utilized the $\mathrm{R}$ and $\mathrm{B}$ bands. On the basis of the classification results, all the virtual images were able to detect changes in color, although the results obtained with samples stored at $10^{\circ} \mathrm{C}$ were not as accurate compared to those obtained with samples stored at $4{ }^{\circ} \mathrm{C}$. In both cases, samples were classified into two deterioration reference classes (DRCs): Class A and Class B, which included the majority of the samples analyzed on the first days and on the last days of storage, respectively. The virtual images based on the $\mathrm{R}$ and $\mathrm{B}$ spectral ranges gave better results in the internal validation procedure than those based on IR and R bands. In all cases, Class B had increased lightness and $a^{*}$ values compared to Class $A$, but image combination based on $B / R$ and $R-B / R+B$ was highly sensitive and best reflected the change in colors associated with discoloration. Taken together, these results confirmed that the $\mathrm{R}$ and $\mathrm{B}$ spectral ranges contain enough information for segregating extreme stages of discoloration or deterioration. This method could be used to detect the most significant color changes in RTU packed spinach leaves under different refrigeration conditions. Additionally, this method allows for a more spatially detailed determination compared to other colorimetric techniques that analyze only a small portion of a sample and can lead to errors and inaccurate results if the analysis is not repeated in different zones on the sample surface. Moreover, colorimetric measurements can only be made if contact can be made with the surface of the leaves, and the process cannot be automated. However, analyzing the histogram for the whole image of each sample is an easier and faster technique that allows quantification based on the original colors of the sample. Further research should be conducted to improve the performance of the classification models with external validations, adding additional wavelengths to generate the virtual images.

\section{Acknowledgements}

This research was conducted at the Universidad Politécnica de Madrid (Spain) and was supported by the project MULTIHORT (AGL2008-05666-C02-01/ALI), funded by the Spanish Ministerio de Ciencia e Innovación (MCINN) and by the European project ISAFRUIT (FP6 FOOD 016279-2). The authors wish to acknowledge Kernel Export s.l. for storing and providing the vegetal material used in this work.

\section{References}

Abbott, J.A., 1999. Quality measurement of fruits and vegetables. Postharvest Biol. Technol. 15, 207-225.

Allende, A., Luo, Y., McEvoy, J.L., Artés, F., Wang, C.Y., 2004. Microbial and quality changes in minimally processed baby spinach leaves stored under super atmospheric oxygen and modified atmosphere conditions. Post harvest Biol. Technol. $33,51-59$.

Bodria, L., Fiala, M., Guidetti, R., Oberti, R., 2004. Optical techniques to estimate the ripeness of red-pigmented fruit. Trans. ASAE 47, 815-820.

Borhan, M.S., Panigrahi, S., Lorenzen, J.H., Gu, H., 2004. Multispectral and color imaging techniques for nitrate and chlorophyll determination of potato leaves in a controlled environment. Trans. ASAE 2, 599-608.

Coles, G.D., Peter, J., Ammerink, J., Wallace, A.R., 1993. Estimating potato crisp colour variability using image analysis and a quick visual method. Potato Res. 32, $127-134$.

Ferrante, A., Incrocci, L., Maggini, R., Serra, G., Tognoni, F., 2004. Colour changes of fresh-cut leafy vegetables during storage. J. Food Agric. Environ. 2, 40-44.

Francis, F.J., Clydesdale, F.M., 1975. Food Colorimetry: Theory and Applications. AVI Publ. Co., Westport.

Gitelson, A.A., Merzlyak, M.N., 1996. Signature analysis of leaf reflectance spectra: algorithm development for remote sensing of chlorophyll. J. Plant Physiol. 148, 494-500.

Hahn, F., 2002. AE-automation and emerging technologies: multi-spectral prediction of unripe tomatoes. Biosyst. Eng. 81, 147-155.

Herrero, A., Lunadei, L., Lleó, L., Diezma, B., Ruiz-Altisent, M., 2011. Multispectral vision for monitoring fruit ripeness. J. Food Sci. 76, E178-E187.

Jacxsens, L., Devlieghere, F., Debevere, J., 2002. Temperature dependence of shelflife as affected by microbial proliferation and sensory quality of equilibrium modified atmosphere packaged fresh produce. Postharvest Biol. Technol. 26, $59-73$.

Kader, A.A., 2002. Quality parameters of fresh-cut fruit and vegetable products. In: Lamikanra, O. (Ed.), Fresh-cut Fruits and Vegetables. CRC Press, Boca Raton, FL.

Kleynen, O., Leemans, V., Destain, M.F., 2005. Development of a multi-spectral vision system for the detection of defects on apples. J. Food Eng. 69, 41-49.

Klockow, P.A., Keener, K.M., 2009. Safety and quality assessment of packaged spinach treated with a novel ozone-generation system. LWT Food Sci. Technol. 42, $1047-1053$.

Kranzier, G.A., 1985. Applying digital image processing in agriculture. Agric. Eng. A $66,11-13$.

Lunadei, L., Galleguillos, P., Diezma, B., Lleó, L., Ruiz-Garcia, L., 2011. A multispectral vision system to evaluate enzymatic browning in fresh-cut apple slices. Postharvest Biol. Technol. 60, 225-234.

Lleó, L., Barreiro, P., Ruiz-Altisent, M., Негrero, A., 2009. Multispectral images of peach related to firmness and maturity at harvest. J. Food Eng. 93, 229-235.

Marcus, R.T., Kurt, N., 1998. Chapter 2 The Measurement of Color. Azimuth, NorthHolland, pp. 31-96. 
Otsu, N. 1979. A threshold selection method from gray-level histograms. IEEETrans. Syst. Man Cybern. 9, 62-66

Otto, M., 2007. Chemometrics: Statistics and computer application in analytical hemistry. Weinheim: Wiley-VCH-Verl.

Park, B., Chen, Y.R., 2001. AE-automation and emerging technologies: co-occurrence matrix texture features of multi-spectral images on poultry carcasses. J. Agric. Eng. Res. 78, 127-139.

Ragaert, P., Verbeke, W., Devlieghere, F., Debevere, J., 2004. Consumer perception and choice of minimally processed vegetables and packaged fruits. Food Qual. Preference 15, 259-270.

Sims, D.A., Gamon, J.A., 2002. Relationships between leaf pigment content and spec tral reflectance across a wide range of species, leaf structures and developmenta stages. Remote Sens. Environ. 81, 337-354.

Studman, C.J., 2001. Computers and elect ronics in postharvest technology-a review. Comput. Electron. Agric. 30, 109-124.

Tunde, V., Feldoldi, J., 2000. Enhancing colour differences in images of diseased mushrooms. Comput. Electron. Agric. 26, 187-198.

Ward Jr., J.H., 1963. Hierarchical grouping to optimize an objective function. J. Am. Stat. Assoc. 58, 236-244.
Watada AE Oi L 1999. Quality of fresh-cut produce Postharvest Biol. Technol. 15 201-205.

Xue, L., Yang, L., 2009. Deriving leaf chlorophyll content of green-leafy vegetables from hyperspectral reflectance ISPRS I. Photogramm. Remote Sens.64.97-106.

Yam, K.L., Papadakis, S.E., 2004. A simple digital imaging method for measuring and analyzing color of food surfaces. J. Food Eng. 61, 137-142.

Zheng, C., Sun, D.-W., Zheng, L., 2006a. Recent applications of image texture for evaluation of food qualities-a review. Trends Food Sci. Technol, 17 $113-128$.

Zheng, C., Sun, D.-W., Zheng, L., 2006b. Recent developments and applications of image features for food quality evaluation and inspection-a review. Trends Food Sci. Technol. 17, 642-655.

Zhou, T., Harrison, A.D., McKellar, R., Young, J.C., Odumeru, J., Piyasend, P., Lu, X. Mercer, D.G. Karr, S. 2004. Determination of acceptability and shelf life of ready-to-use lettuce by digital image analysis. Food Res. Int. 37 $875-881$.

Zude, M., 2003. Comparison of indices and multivariate models to non-destructively predict the fruit chlorophyll by means of visible spectrometry in apple fruit. Anal. Chim. Acta 481, 119-126. 